\title{
五味子三萜天然产物的合成进展
}

\author{
郭 璐汤平平* \\ (南开大学化学学院 元素有机化学国家重点实验室 天津 300071)
}

\begin{abstract}
摘要 五味子类植物在我国分布广泛, 作为传统中药具有抗肝炎等生物活性. 同时该类天然产物具有独特的多种骨架 结构, 因此引起广大合成化学家的关注. 对近期相关五味子三萜类天然产物的全合成进行了综述.

关键词 五味子科; 五味子三萜; 全合成
\end{abstract}

\section{Recent Advance in the Synthesis of Natural Products of Schisandra Triterpenoid}

\author{
Guo, Lu Tang, Pingping* \\ (State Key Laboratory of Elemento-organic Chemistry, College of Chemistry, Nankai University, Tianjin 300071)
}

\begin{abstract}
Schisandraceae is widely spread within our country. As a kind of traditional Chinese herb, it is known for its anti-hepatitis bioactivity. The diversified and unique structures in this family have drawn the attention of many synthetic chemists. The recent efforts towards the total synthesis of schisandra triterpenoid are summarized.

Keywords schisandraceae; schisandra triterpenoid; total synthesis
\end{abstract}

五味子科 (Schisandraceae) 植物包含五味子属 (Schisandra) 和南五味子属(Kadsura), 世界上有五十余 种，主要分布在东南亚和北美. 我国有 29 种, 独有 13 种，主要分布在西南地区，其中最有名的五味子 (Schisandra chinensis)广泛分布于我国东北.

自 1973 年日本的 Yoshikoshi 课题组首次分离纯化 出单体五味子三萜类化合物以来, 已经有四百余种 ${ }^{[1-3]}$ 五味子三萜类化合物被提取分离出来. 这些化合物大多 具有抗肝炎、抗肿瘤、抗 HIV 等活性. 其独特的骨架结 构和良好的生物活性吸引了众多化学家进行研究. 2005 年, 杨震课题组首次报道了关于 micrandilactone A 的合 成 ${ }^{[3]}$, 后续有多个小组对该家族天然产物的合成进行了 研究，实现了不同结构类型化合物的全合成.

\section{1 五味子三萜类化合物的结构与生物活性}

五味子的干燥成熟果实 ${ }^{[4]}$, 在中医中常用于治疗肝 炎, 除此之外部分五味子还被用做食品和饮料的原料 ${ }^{[1]}$. 近年来, 我国科研人员对五味子的茎叶中分离得到的新
型结构进行研究, 发现许多新型五味子化合物具有抗艾 滋病病毒的活性 ${ }^{[4]}$.

2003 年, 孙汉董课题组 ${ }^{[5]}$ 首次通过 X 射线衍射, 分 析确定了该家族 micrandilactone A 的结构. 之后近二十 年，该小组又从五味子科植物中分离得到 100 余种化合 物 ${ }^{[1-2]}$. 孙汉董课题组等根据碳骨架结构的不同, 将其分 为三类, 如 Scheme 1 所示, 分别为羊毛甾烷类 (Lanostane)、环菠萝烷类(Cycloartane) 以及五味子降三萜类 (Schisandra nortriterpenoids). 其中根据骨架的不同氧化 形式和结构特征，又进一步被细分为不同类型. Schiartane 型作为五味子降三萜类化合物中最基本的骨架类 型, C18 位发生降解后得到 18-norschiartane 型, 进行 $\mathrm{C} 13-\mathrm{C} 14$ 碳碳键断裂和 $\mathrm{C} 13-\mathrm{C} 16$ 位环合得到 preschisanartane 型, 具有 7/8/3 稠合碳环.

\section{2 五味子三萜的合成研究}

随着五味子三萜类化合物被不断分离, 关于五味子 三萜类化合物的不同合成策略和方法也陆续涌现. 杨震

* Corresponding author. E-mail: ptang@nankai.edu.cn

Received May 28, 2021; revised July 15, 2021; published online August 17, 2021.

Dedicated to the 100th anniversary of Chemistry at Nankai University.

Project supported by the National Key Research and Development Program of China (No. 2016YFA0602900), the National Natural Science Foundation of China (Nos. 21672110, 21925105), and the Natural Science Foundation of Tianjin City (No. 18JCJQJC47000).

国家重点研发计划(No. 2016YFA0602900)、国家自然科学基金(Nos. 21672110, 21925105)、天津市自然科学基金(No. 18JCJQJC47000)资助项目. 


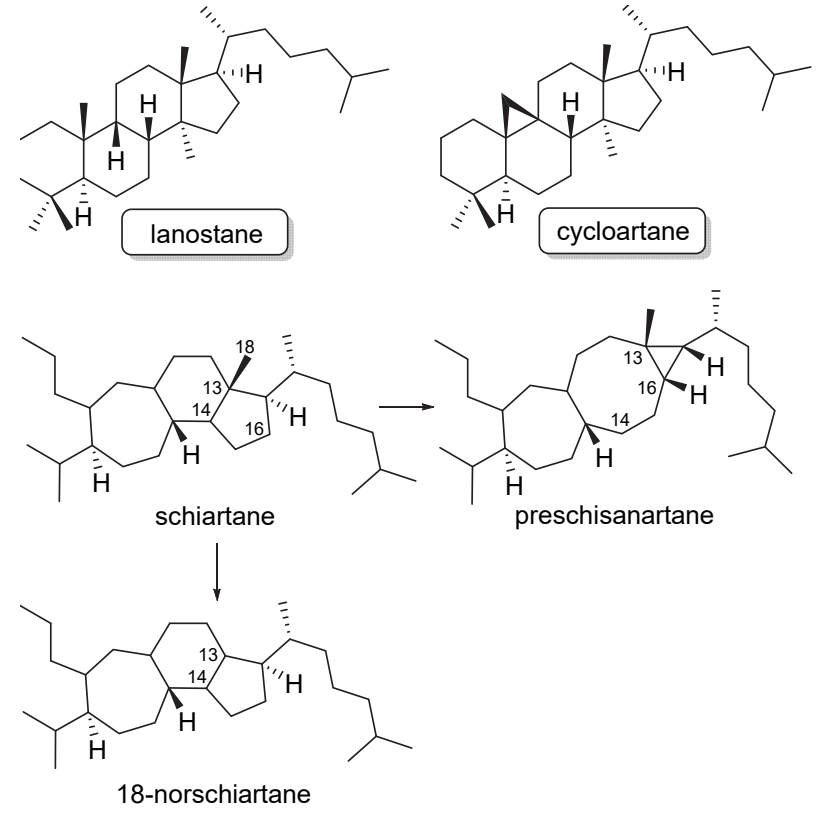

Schisandra nortriterpenoid

图式 1 五味子三萜类化合物基本骨架

Scheme 1 Basic structure of Schisandra triterpenoid natural products

小组 ${ }^{[3,6-7]}$ 在 2005 年通过发展硫脲-钴催化的 PausonKhand 反应，构建了 micrandilactone A 的 F、G、H 环，并 成功应用在后续五味子降三萜的全合成中. 2006 年, 通 过烯烃关环复分解构建七元环，又完成了该分子 A、B、 C 三环的构建. 2008 年, 该小组利用 Claisen 重排构建了 该分子的 D、E 环. 2006 年, Radhakrishnan 小组 ${ }^{[8]}$ 通过 [6+3]环加成实现了该类天然产物 5/8 并环结构及氧桥 的构建. 2007 年, Theodorakis 小组 ${ }^{[9]}$ 利用酸介导的扩环 反应实现了 lancifodilactone $\mathrm{F}$ 中 $\mathrm{A} 、 \mathrm{~B}$ 环及其他含有 $3 / 6$ 并环骨架结构的构建. 2008 年, 陈绰小组 ${ }^{[10]}$ 利用两次环 加成分别构建了全碳季碳手性中心及六、七元环, 实现 了 lancifodilactone F 核心骨架的构建. 2008 年, Paquette 小组 ${ }^{[11-13]}$ 报道了 lancifodilactone $G$ 的合成探索, 利用氧 杂 Michael 加成、内酯化反应、烯烃关环复分解构建了 A、B、C 环系，二碘化钐介导的 Pinacol 偶联构建了 C、 D、 $\mathrm{E}$ 环系, 以及分子内立体选择性的自由基环化构建 了包含多个手性中心的 $\mathrm{F}$ 环. Anderson 小组 ${ }^{[14-16]}$ 则于 2008 年和 2012 年采用钯催化的串联环化反应构建了含 芳环结构的 rubriflordilactone A 的 C、D、E 环系, 2014 年则利用内酯化/氧杂 Michael加成构建了五味子降三萜 骨架的核心 A、B 环. Mehta 小组 ${ }^{[17-20]}$ 分别于 2009 年和 2010 年采用 Morita-Baylis-Hillman 反应引入侧链, 并通 过 Michael 加成反应快速构建了五味子三萜化合物的 A、B、C 环和 F、G、H 环. 之后 2013 年, 该小组从 Hajos-Parrish 酮出发快速构建了 A、B、C、D 环系. Ghosh
小组 ${ }^{[21-23]}$ 在 2010 年利用烯烃关环复分解构建了 schintrilactones 的 A、B、C 环，利用烯烃开环复分解 (ROM)-关环复分解(RCM)串联构建了 schintrilactones 的 C、D、E 环系, 2011 年利用分子内的 Diels-Alder 反应构 建了 rubriflordilactone B 的 C、D 环系. 2012 年, Parrain 小组 ${ }^{[24]}$ 利用钯催化的 Sonogashira 偶联-内酯化-Cope 重 排串联反应构建了 lancifodilactone $\mathrm{F}$ 及 micrandilactone B 的 A、B、C 环. 2015 年彭羽小组 ${ }^{[25]}$ 利用自由基的 1,5氢篗取/环化串联反应，以及 Mukaiyama Aldol 反应成功 构建了 rubriflordilactone B 的 D、E、F、G 环. 谢志翔 小组 ${ }^{[26-27]}$ 先后于 2016 年和 2017 年利用手性氨基酸催化 的不对称 Mukaiyama-Michael 反应、[2+2+2]环加成反 应以及基于自由基-偶极交叉环加成(PRCC)构建了 rubriflordilactone B 的 C、D、E 环和 D、E、F、G 环. 2017 年, Kalesse 小组 ${ }^{[28]}$ 利用 Pinacol 偶联构建了 schigulautone $\mathrm{A}$ 的九元环. 以上片段的合成探索, 为该家族的全合成 提供了宝贵经验.

自 2011 年杨震小组报道了首例五味子三萜化合物 $( \pm)$-schindilactone $\mathrm{A}$ 的全合成以来 ${ }^{[29-32]}$, 该小组先后完 成了 propindilactone $\mathrm{G}^{[33]}$ 的不对称全合成, 以及 19dehydroxyl arisandilactone $\mathrm{A}^{[34]}$ 、 lancifodilactone $\mathrm{G}$ acetate ${ }^{[35]}$ 和 pre-schisanartanin $\mathrm{C}^{[36]}$ 的全合成. 近期，桂敬汉 小组 ${ }^{[37-38]}$ 也完成了 propindilactone $G$ 的全合成. 李昂小 组 ${ }^{[39-40]}$ 于 2014 年和 2016 年先后完成了 rubriflordilactone A 和 rubriflordilactone B 的不对称全合成. Anderson 小组 也于 2015 年和 2019 年先后实现了 rubriflordilactone $\mathrm{A}^{[41]}$ 和 rubriflordilactone $\mathrm{B}^{[42]}$ 的不对称全合成. 丁寒锋小 组 ${ }^{[43]} 2018$ 年完成了首个含羊毛甾烷骨架 atropschiglautone A 的全合成. 本小组 ${ }^{[44-46]}$ 也于 2015 年和 2017 年先后完成了 schilancitrilactone A、schilancitrilactones B 和 C、schilancidilactones A 和 B 以及差向异构 体 20-epi-schilancitrilactone A 的全合成. 其中, 2018 年以 前的全合成工作已有很全面的综述 ${ }^{[47-49]}$, 在此不再赘 述. 本文对近期五味子三萜化合物的全合成按发表年份 先后顺序进行综述 ${ }^{[50-51]}$.

\section{1 丁寒锋小组对 atrop-schiglautone A 的全合成}

Schiglautone A 由阮汉利等 ${ }^{[52] 于 ~} 2011$ 年从 S. glaucescens 中分离得到, 具有新型羊毛甾烷骨架结构. 该化 合物含有 $6 / 7 / 9$ 稠合环系以及独特的桥头双键; 共有 7 个手性中心, 包括 3 个季碳手性中心. 丁寒锋小组 ${ }^{[43]}$ 从 原料 17 出发, 实现了 schiglautone A 的阻转异构体的全 合成. 其关键转化包括: (1)钛催化的自由基环化反应构 建反式稠合 6/7 环系; (2)关环复分解和 Claisen 重排构建 含张力九元环; (3)底物控制的 Michael 加成立体选择性 引入 $\mathrm{C}-17$ 位侧链. 
<smiles>C=CC1=CC[C@@H]2C[C@]3(OC(=O)C[C@@H]3O[C@@H]2C)[C@]1(Br)OBr</smiles>

$A B C$ ring of micrandilactone $A$ Yang Z. 2006<smiles>C[C@H]1C(=O)CC[C@]23CC2C(=O)CC[C@]13C</smiles>

key precursor of schisanartanes Theodorakis 2007

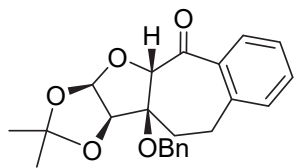

$C D$ ring unit of rubrifloradilactone $\mathrm{B}$ Ghosh 2011

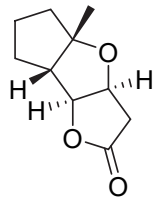

FGH tricyclic ring of rubrifloradilactone $\mathrm{C}$ Mehta 2010

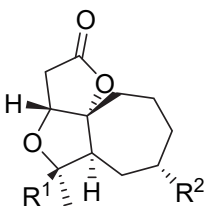<smiles>CC1C2=CCCCCC23OC(=O)C[C@H]1O3</smiles>

$A B C$ tricyclic ring of schisanartanes Paquette 2008; Ghosh 2010; Mehta 2010<smiles>[R]C1C([R])(C)[C@H]2CC(=O)O[C@]12[R]</smiles><smiles>O=C1C[C@H]2OC[C@H]3C=CC4CCC[C@H](O)C4=C[C@]32O1</smiles>

$A B$ ring Mehta 2009; Ghosh 2011; Anderson 2014

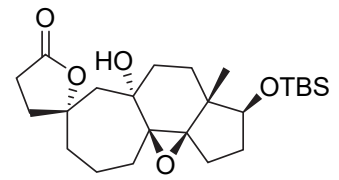

$A B C$ and $A B C D$ ring of the shiartane-type nortriterpenoids Parrain 2012; Mehta 2013<smiles>CC1(C)C2=C(CC=C2)[C@H]2C[C@H]1C=CC(=O)[C@H]2O</smiles><smiles>CO[C@]12CC(C)(C)CC[C@]3(CCCCC[C@H]31)O2</smiles>

CDE ring system of lancifodilactone $G$ Radhakrishnan 2006; Paquette 2008<smiles>C[C@H]1CCC=C2CCCCCC2CC1=O</smiles>

$D E$ ring system of micrandilactone $\mathrm{A}$ Yang Z. 2008

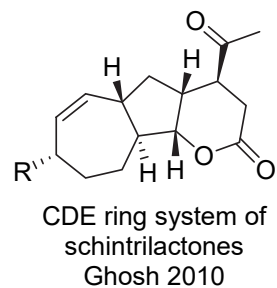
Ghosh 2010

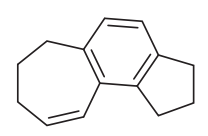

$\mathrm{CDE}$ ring system of rubriflordilactones Anderson 2008 \& 2012

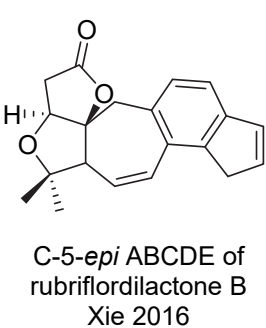

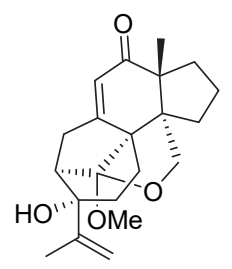

core skeleton of lancifodilactone $\mathrm{F}$

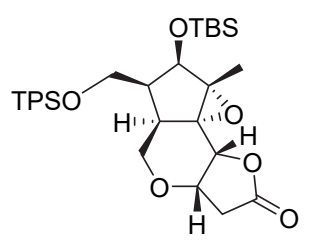

FGH ring of micrandilactone $A \quad F$ ring of lancifodilactone $G$ Yang Z. 2005

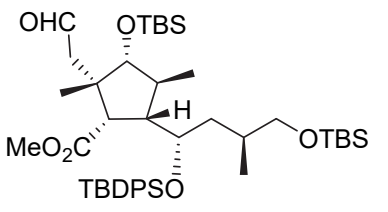

Paquette 2008

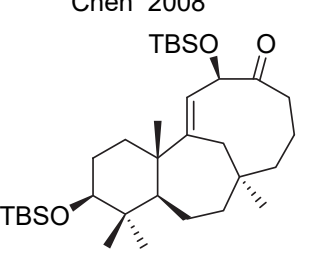

$A B C$ ring of schiglautone $A$ Kalesse 2017

图式 2 五味子三萜类化合物的片段合成

Scheme 2 Segments of synthesis about schisandra triterpenoid natural products

如 Scheme 4 所示, 从反式橙花醇 17 出发, 区域选 择性地环氧化、酰基化得到中间体 18. 18 在钛(III)催化 下发生自由基串联环化, 得到反式 6/7 并环化合物 19. 由于 C-10 位甲基位阻, 19 的双键顺利发生立体选择性 的环氧化得到 20. 20 经过 Ley-Griffith 氧化和 MeerweinPonndorf-Verley 还原, 实现了 C-3 位羟基的手性翻转, 茮基对其进行保护后得到 21. 随后 21 再次在钛催化下, 环氧开环后同丙烯酸甲酯加成, 接着发生酯交换, 以 91\%收率得到六元环内酯化合物 22. 紧接着 22 的内酯 环被氢化铝锂还原, 得到的两个羟基经过 Swern 氧化转 化为醛基. 之后乙烯基格氏试剂与醛基加成, 并用三甲 基硅基保护, 以 $1: 1$ 比例得到一对非对映异构体. 23 再 在强碱下与烯丙基碘反应得到氧烷基化产物, 随后在 $120{ }^{\circ} \mathrm{C}$ 下发生 Claisen 重排得到双烯化合物 24. 24 发生 烯烃关环复分解反应, 实现了九元环的构建, 得到三环 产物 25. 25 在 Jones 氧化后与甲基锂发生 1,2 加成, 所得
到的烯丙醇再通过 Dauben-Michno 氧化, 得到不饱和酮 26. 26 在溴化亚铜二甲硫醚络合物的作用下，与格氏试 剂 27 进行 Michael 加成, 用三乙基氯硅烷生成烯醇硅醚, 再用甲基二环氧乙烷(DMDO)对两个双键进行氧化，生 成不稳定的 $\alpha$-羟基酮 28.28 通过氯铬酸吡啶盐氧化, 经 由环状铬酸酯中间体实现环氧断裂, 得到醛 30 . 醛 30 通过 Horner-Wadsworth- Emmons 反应得到 $Z$ 式烯烃 32. 随后 32 经过碘代与氧化消除, 实现了环外双键的构建. 最终 33 经过氧化脱去 C-3 位羟基的苄基保护，以及 Luche 还原, 得到 C-12 位羟基产物 34. 34 用四丁基氟化 铵脱去硅基保护，从而实现了 atrop-schiglautone A 的全 合成.

\subsection{Anderson 小组对( - -rubriflordilactone $B$ 和} (一)-pseudo-rubriflordilactone B 的全合成

18-norschiartane 型 rubriflordilactone B 由孙汉董等 于 2006 年从红花五味子 S. rubriflora 中分离得到 ${ }^{[53]}$, 具 


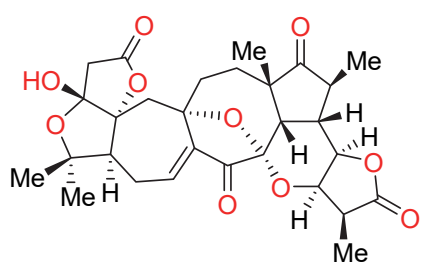

Schindilactone A (1)

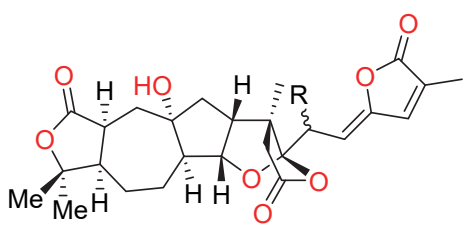

Schilancitrilactone $\mathrm{B}(\mathrm{R}=\alpha$-Me $)(4)$ Schilancitrilactone $\mathrm{C}(\mathrm{R}=\beta$-Me $)(5)$

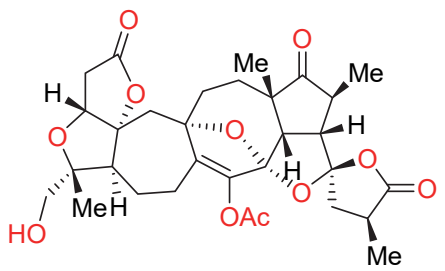

lancifodilactone G Acetate (9)

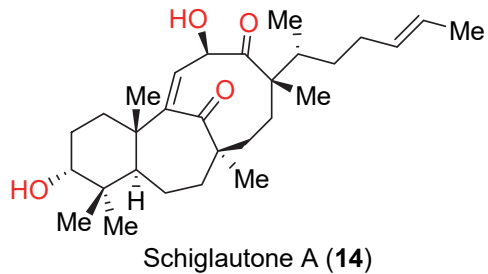

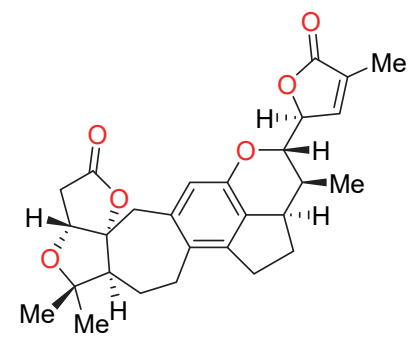

Rubriflordilactone A (2)

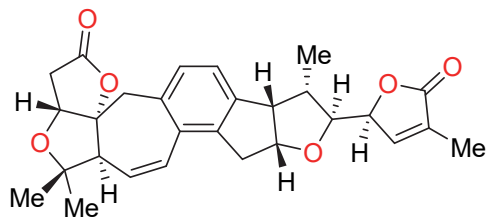

Rubriflordilactone B (6)

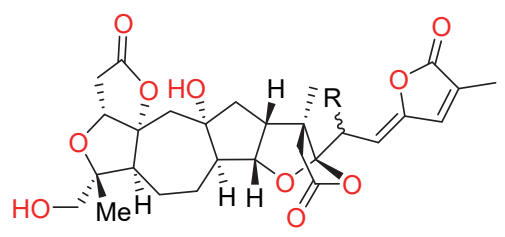

Schilancitrilactone $\mathrm{A}(\mathrm{R}=\alpha-\mathrm{Me})(10)$ 20-epi-Schilancitrilactone $\mathrm{A}(\mathrm{R}=\beta-\mathrm{Me})(11)$

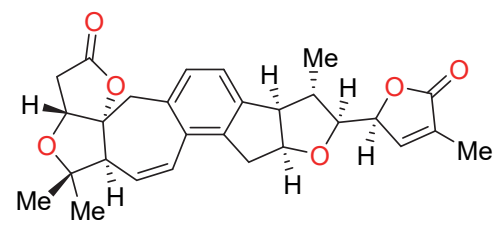

Pseudo-rubriflordilactone B (15)

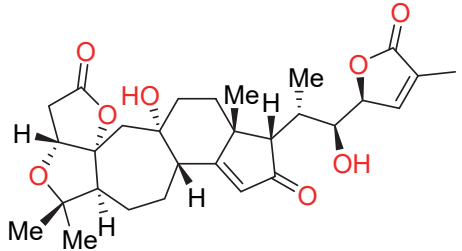

Propindilactone G (3)

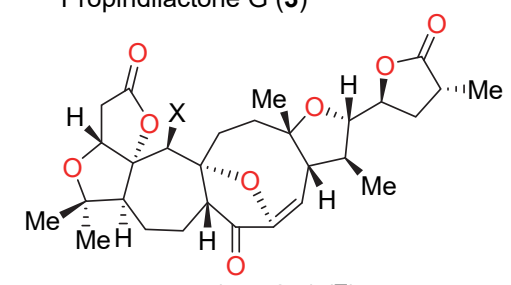

Arisandilactone $\mathrm{A}(\mathrm{X}=\mathrm{OH})(7)$ 19-Dehydroxyl arisandilactone $A(X=H)(8)$

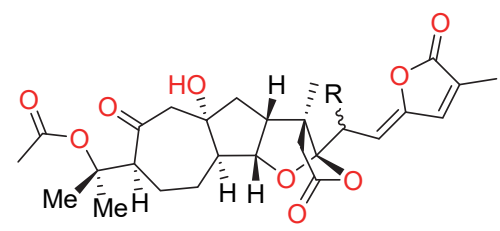

Schilancidilactone $\mathrm{A}(\mathrm{R}=\alpha-\mathrm{Me})(12)$ Schilancidilactone $\mathrm{B}(\mathrm{R}=\beta \mathrm{Me})(13)$

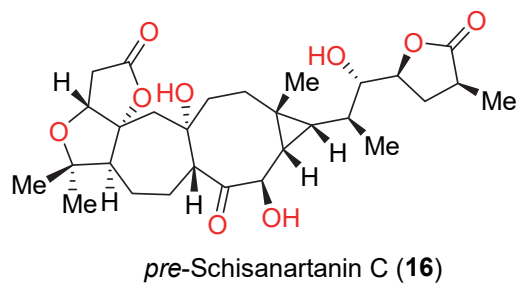

图式 3 五味子三萜类化合物的全合成

Scheme 3 Total synthesis of Schisandra triterpenoid natural products

有独特芳构化 D 环结构, 共有 7 个环系, 包括 1 个四取 代苯环及 8 个手性中心. 李昂小组 ${ }^{[39,40]}$ 采用 $6 \pi$ 电环化 先后于 2014 年和 2016 年分别完成了 rubriflordilactone A 和 rubriflordilactone B 的全合成. 由于 rubriflordi- lactones B 的合成结构与之前分离提出的结构 NMR 数据不 同, 近期, 李昂小组通过化学合成确定了晶体数据和 NMR 数据的对应结构 ${ }^{[54]}$. 而与此同时 Anderson 小组 ${ }^{[42]}$ 也通过化学合成的方法对 rubriflordilactone B 和 pseudo-rubriflordilactone B 的结构进行了进一步验证, 其关键转化包括: (1)后期 Nozaki-Hiyama-Kishi 偶联实 现片段连接; (2)铑催化 $[2+2+2]$ 炔烃三聚化反应构建 芳香 D 环. Anderson 小组在之前对 rubriflordilactone A 的 合成 ${ }^{[41]}$ 中就采用了钯或钴催化环化构建 CDE 环系.

如 Scheme 5 所示, 先构建 A/B 环系, 即 rubriflordilactone A 合成中的相同片段 $\mathbf{4 1}$. 从不饱和酯 35 出发, 在 铜催化下与格氏试剂发生加成反应, 酯基被二异丁基氢 化铝还原得到伯醇, 随后加成得到的双键经过 Sharpless
不对称环氧化得到 36. 烯丙基格氏试剂进攻 36 中的环 氧, 氧化伯醇至羧酸, 得到 $\beta$-内酯 37. $\beta$-内酯 37 与甲基 格氏试剂加成得到含偕二甲基叔醇 $\mathbf{3 8} .38$ 末端双键被氧 化切断，在酸作用下生成缩醛，同时脱除伯醇硅基保护, 进一步氧化为醛 39. 通过 Horner-Wadsworth-Emmons 反 应将 39 转化为烯烃, 接着在三氟乙酸作用下脱去缩醛 甲基保护并发生酯交换. 半缩醛 $\mathbf{4 0}$ 进一步水解，得到的 羟基与五元环不饱和酯发生氧杂 Michael 加成, 同时脱 除端炔硅基保护, 得到片段 41. 之后, 分别合成 C-16、 $\mathrm{C}-17$ 手性不同的 4 个片段. 从简单醛 $\mathbf{4 2}$ 出发, 与原位生 成的烯酮发生 $[2+2]$ 环加成得到 $\beta$-内酯 44 , 之后同烯丙 醇发生酯交换得到 45.45 经过 Ireland-Claisen 重排生成 羧酸, 与三甲硅基重氮甲烷作用得到甲酯 46. 氢化铝锂 还原 46 酯基到伯醇, 并用硅基进行保护. 氧化切断烯 烃，分子内环化生成甲缩醛，并脱除硅基保护得到 47 . 伯醇 47 经过氧化、烯基化、消除得到端炔并保护，引入 第一个炔基侧链, 然后氧化脱除另一侧链保护基, 再氧 
<smiles>[R]C(=O)Oc1ccc(C(F)(F)F)cc1</smiles>

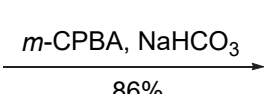

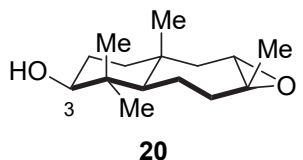

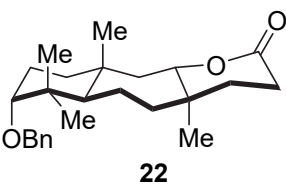

(1) $\mathrm{LiAlH}_{4}$, then DMSO $(\mathrm{COCl})_{2}, \mathrm{NEt}_{3}$

(2) vinylMgBr, TMSCI $67 \%$, 2 steps, $1: 1$ d.r.

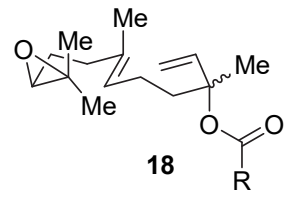

$\mathrm{Cp}_{2} \mathrm{TiCl}_{2}, \mathrm{Mn}$ 2,4,6-colidine, TMSCl $75 \%, 53-g$ scale

(1) TPAP, NMO then $\mathrm{Al}(i-\mathrm{OPr})_{3}$ $72 \%, 5: 1$ d.r.

(2) $\mathrm{NaH}, \mathrm{BnBr}, 93 \%$

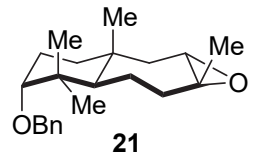

$\mathrm{Cp}_{2} \mathrm{TiCl}_{2}, \mathrm{Zn}$ 2,4,6-colidine $\cdot \mathrm{HCl}$ Methyl acrylate, $91 \%$

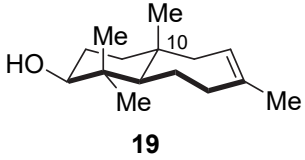

19

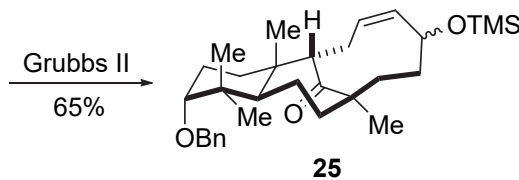

25
(1) $\mathrm{CrO}_{3}, \mathrm{H}_{2} \mathrm{SO}_{4}$

(2) $\mathrm{MeLi}$; then $\mathrm{PDC}, \mathrm{SiO}_{2}$ $60 \%, 2$ steps

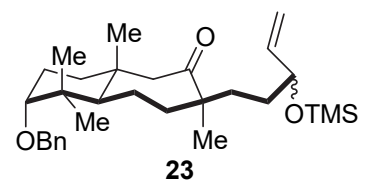

\section{LiHMDS allyl iodide, $-40^{\circ} \mathrm{C}$ $120^{\circ} \mathrm{C}, 85 \%$}

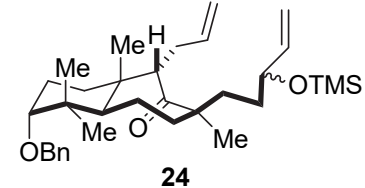

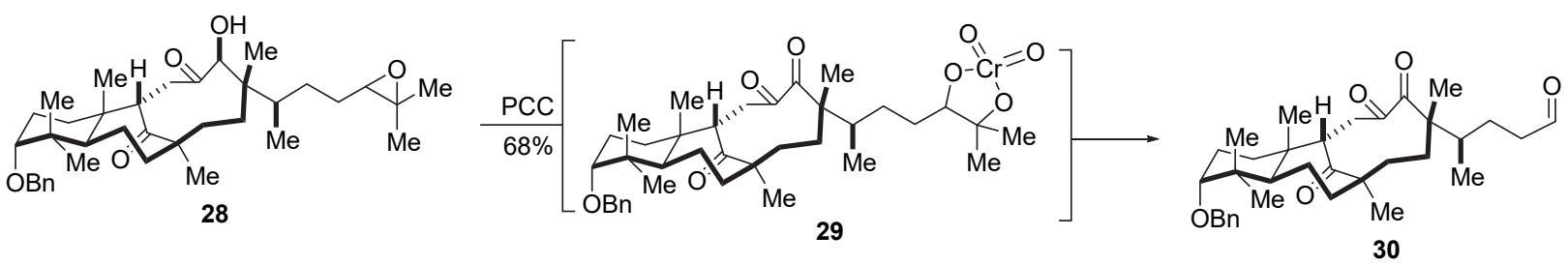

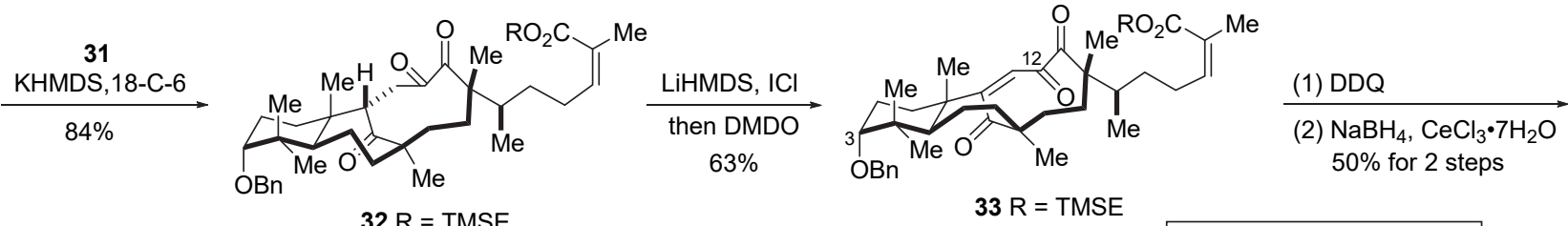

$32 \mathrm{R}=\mathrm{TMSE}$

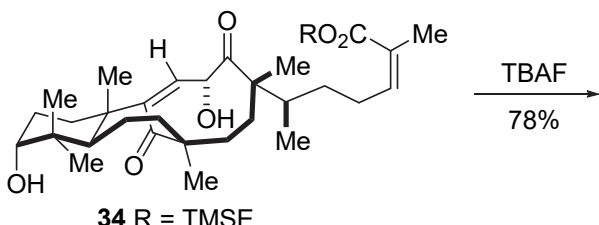

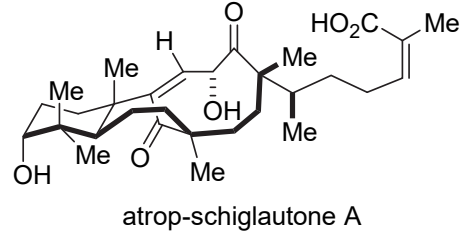

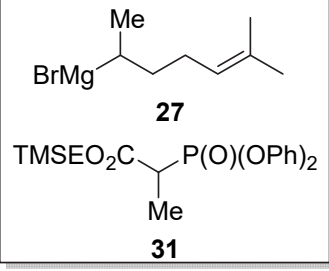

图式 4 丁寒锋小组对 atrop-schiglautone A 的全合成

Scheme 4 Ding's total synthesis of atrop-schiglautone A

化、炔基化, 引入第二个炔基侧链得到 48. 将 48 缩醛保 护基由甲基转换为乙酰基, 然后与碘、吗啡啉作用生成 炔基碘 49. 在 Lewis 酸三氟甲磺酸铋的促进下，与呋喃 片段 50 发生加成, 完成 $\mathrm{F} 、 \mathrm{G}$ 环构建, 得到片段 51a 和 51b. 片段 59a 从 $\beta$-差基酯 52 出发, 在二异丙基氨基锂 (LDA)作用下烯醇化, 同烯丙基碘作用得到 53. 采用之 前同样转化得到五元环内酯 54. 受底物控制, 54 在 LDA 作用下与碘甲烷反应, 以 $3: 1$ 的 $d r$ 值引入甲基. 五元 环内酯 55 经过还原, 甲基保护缩醛, 脱除伯醇硅基保
护, 得到与 47 的 C-16、C-17 手性相反的中间体 56. 之 后，采用同样方法引入两个炔基侧链，但手性无法控制， 得到四个偶联前体 59a 59d. 其中, 59b 和 59c 可分离得 到单一异构体, 59a 和 59d 不可分离. 基于 Anderson 课 题组之前工作, 将炔基碘 51a、59a/59d 与醛 41 分别进 行加成, 之后脱除硅基保护得到三炔 60、61. 在铑催化 下, 60 和 61 分别发生 $[2+2+2]$ 环化构建了芳环，接着用 对甲苯磺酸进行了脱水消除, 从而实现了 (一)-rubriflordilactone $\mathrm{B}$ 和( - -) pseudo-rubriflordilactone $\mathrm{B}$ 的全合成. 

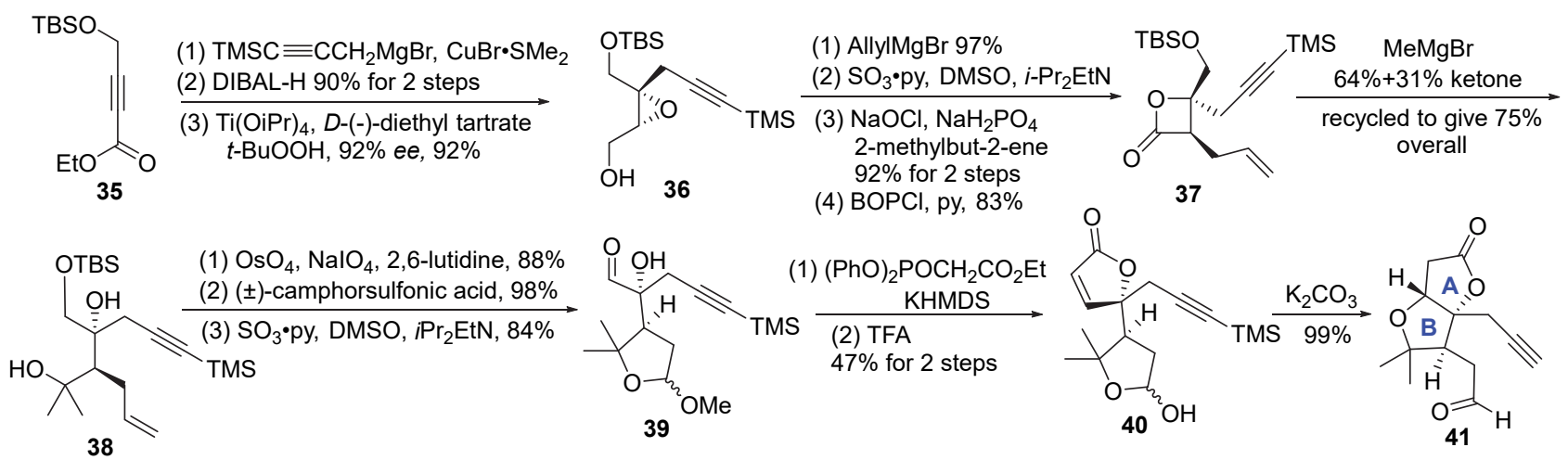

(4) $\mathrm{BOPCl}, \mathrm{py}, 83 \%$

37

(1) $(\mathrm{PhO})_{2} \mathrm{POCH}_{2} \mathrm{CO}_{2} \mathrm{Et}$

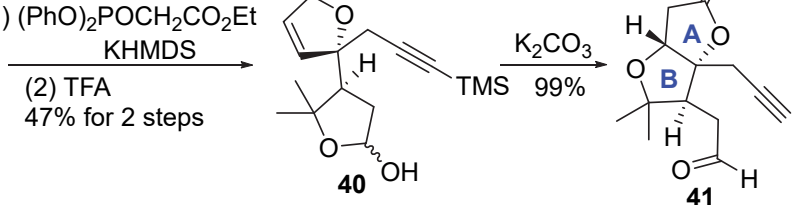

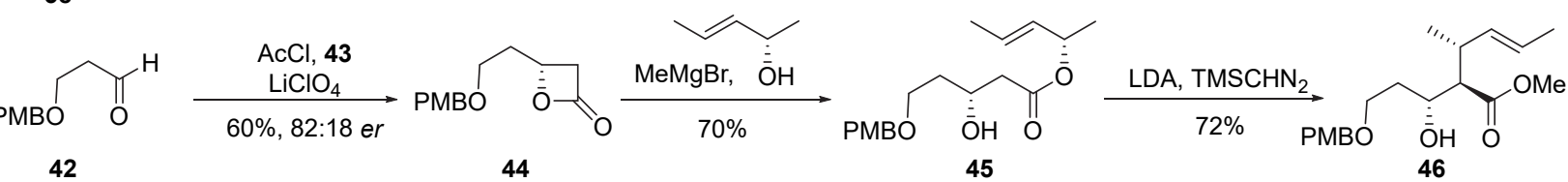

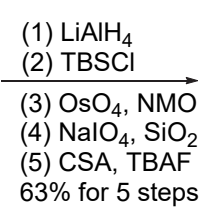

$63 \%$ for 5 steps

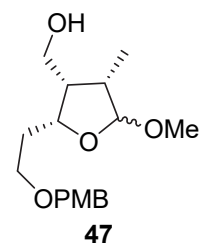

47<smiles></smiles>

$51 \mathrm{a}$
(1) TEMPO, BAIB TMS $\mathrm{PPh}_{3} \mathrm{CH}=\mathrm{CHI}$; NaHMDS, TMSCI (2) DDQ; TEMPO BAIB; $\mathrm{CBr}_{4}, \mathrm{PPh}_{3}$ (3) LiHMDS; $n$-BuLi $59 \%$ over 5 steps<smiles>CC1=C[C@H]([C@H]2O[C@@H](CC#CI)[C@@H](C#CC(C)(C)C)[C@H]2C)OC1=O</smiles>

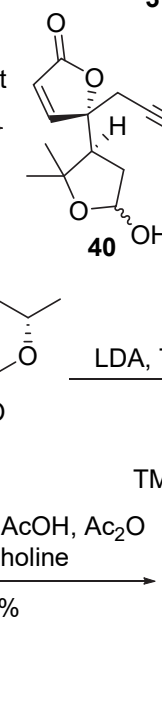

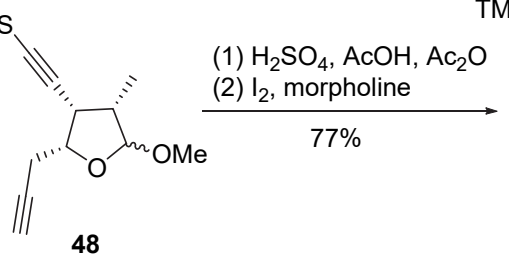

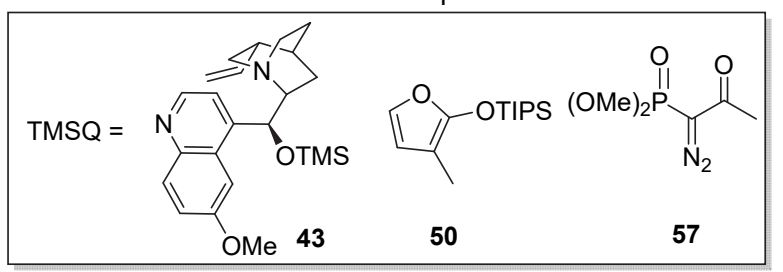

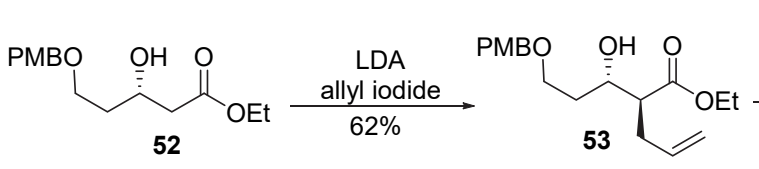

(1) $\mathrm{LiAlH}_{4}$

(2) $\mathrm{TBSCl}$

(3) $\mathrm{OsO}_{4}, \mathrm{NMO}$

(4) $\mathrm{NalO}_{4}, \mathrm{SiO}_{2}$ (5) TPAP, NMO $64 \%$ for 5 steps

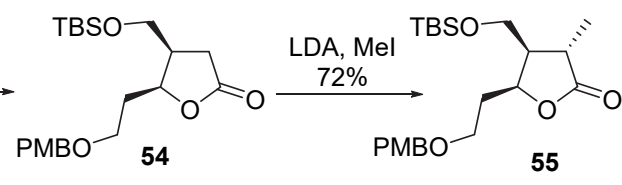

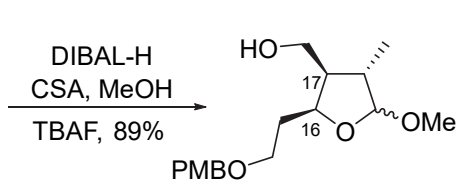

56
(1) 57, TEMPO, BAIB $\mathrm{NaOMe}$ (2) $n$-BuLi, TMSCl (3) DDQ; TEMPO, BAIB; $\mathrm{CBr}_{4}, \mathrm{PPh}_{3}$ LiHMDS; $n$-BuLi $34 \%$ overall

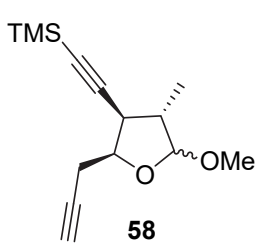

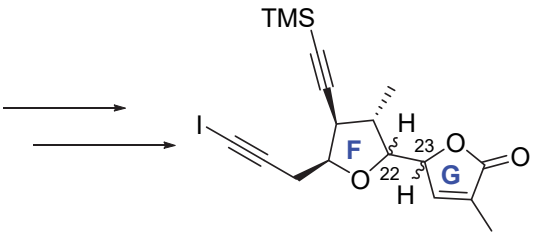

59a: C22S, C23S; 59b: C22S, C23R 59c: $\mathrm{C} 22 R, \mathrm{C} 23 S$; 59d: $\mathrm{C} 22 R, \mathrm{C} 23 R$

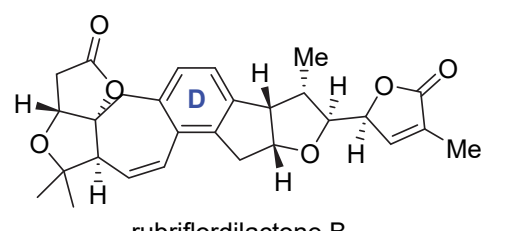

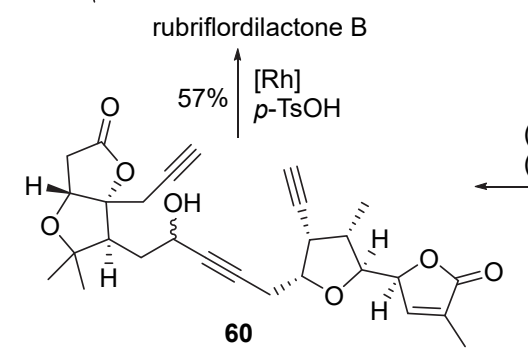

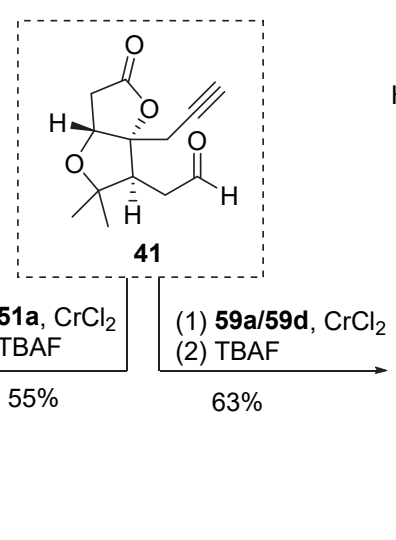

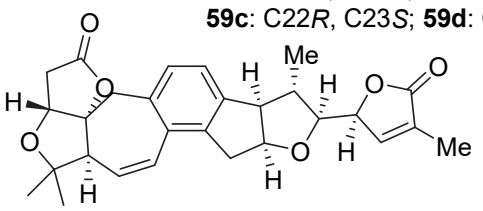

pseudo-rubriflordilactone B

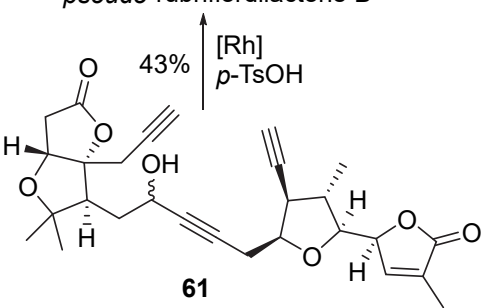

图式 5 Anderson 小组对(一)-rubriflordilactone B 的全合成

Scheme 5 Anderson's total synthsis of (-)-rubriflordilactone B 


\section{3 杨震小组对 pre-schisanartanin C 的全合成}

Preschisanartane 型 pre-schisanartanin C 由孙汉董等 于 2010 年从合荵五味子 S. propinqua var. propinqua 中分 离得到 ${ }^{[55]}$. 不同于该类型其他结构, 该化合物为首例八 元环上不含有氧桥半缩酮的结构, 具有多取代双环 [6.1. $0]$ 壬烷核心结构, 包含 1 个全碳季碳手性中心 C-13, 并 且八元环上含有不稳定的 $\alpha$-羟基酮结构. 杨震小组 ${ }^{[36]}$ 从简单底物 62、63、68 出发, 完成了 pre-schisanartanin $\mathrm{C}$ 的全合成, 并确定了其绝对构型. 关键转化包括: (1) 不对称的 Diels-Alder 反应; (2)金催化的分子内烯炔环化 构建 D-E 环系; (3) $\mathrm{Al}\left(\mathrm{O}^{t} \mathrm{Bu}\right)_{3}$ 异构化构建关键的 $\alpha$-羟基 酮部分; (4) Sharpless 不对称双羟化区域立体选择性地 构建 C-22、C-23 位手性中心.

如 Scheme 6 所示, 采用之前在合成 lancifodilactone $\mathrm{G}$ 时的不对称 Diels-Alder 反应, 用 Corey 催化剂衍生物, 催化烯醇硅醚双烯体 62 与不饱和酩酯亲双烯体 63 反应, 得到六元环化合物 64. 之后, 64 与甲基格氏试剂加成串 联内酯化得到五元环内酯化合物 $\mathbf{6 5}$. 烯醇硅醚 $\mathbf{6 5}$ 进行 环丙烷化后, 在三氯化铁介导下发生氧化碎裂得到不饱 和酮 66. 对不饱和酮 66 进行还原生成烯醇, 与醛发生 Aldol 反应，并保护羟基，得到中间体 67. 同时，由手性 二醇 68 出发, 保护伯醇, 仲醇与锡试剂反应得到 69.69 在正丁基锂引发下发生 Wittig-Still 重排得到伯醇, 随后 进行 Appel 碘代得到片段 70. 将碘代物 70 制成锂试剂, 与 $5 / 7$ 并环中间体 67 进行加成得到 71a 和 71b. 之后混 合物脱去 71 端炔 TMS 保护, 用 DIBAL-H 还原内酯生 成 C-1 位差基, 得到半缩酮中间体 72. 同之前合成策略 不同，在该合成路线中，杨震小组利用内酯环引入 C-10 位羟基. 因此, 72 先与对氟苯甲酰氯反应, 保护炔丙醇 并消除半缩醛裸露差基, 受 C-9 位羟基诱导, 双键环氧 化后水解开环, 立体选择性地引入 C-10 位羟基, 得到化 合物 73. 之后, 氧化半缩醛裸露羟基恢复五元环内酯结 构, 用硅基保护其余羟基. 与之前用烯烃关环复分解构 建八元环不同, 采用金催化, 73 先发生 1,2-迁移生成卡 宾, 再经过分子内环丙烷化构建 D-E 环系, 得到 74 和 75, 中间体 74 可以进一步延长反应时间转化为八元环 化合物 75. 将苯甲酰基烯醇醚转化为甲基烯醇醚 76 后 还原内酯, 与乙酸酐作用生成乙酸酯, 再经过 Mukaiyama Aldol 反应, 立体选择性地引入 C-1 位官能团, 得 到 78. 之后用四氧化锇对 78 进行双羟化得到 $\alpha$-差基酮 79, 再在 $\mathrm{Al}\left(\mathrm{O}^{t} \mathrm{Bu}\right)_{3}$ 下异构化到热力学更稳定化合物 80 . 八元环修饰结束后, 80 伯醇的茮基保护用雷尼镍脱去, 再氧化到醛, 在氯化铬存在下与碘仿反应生成烯基碘, 同锡基试剂 82 发生 Stille 偶联, 引入侧链得到 83.83 脱 去硅基保护, 碱性条件下直接内酯化, 完成 A 环构建,
最后经过 Sharpless 不对称双羟化, 从而实现了 preschisanartanin $\mathrm{C}$ 的全合成.

\section{4 桂敬汉小组对 propindilactone $G$ 的全合成}

Schiartane 型 propindilactone $\mathrm{G}$ 由孙汉董等于 2008 年从合荵五味子 S. propinqua var. propinqua 中分离得 到[56]. 具有独特的 5/5/7/6/5 五环骨架，10 个手性中心, 其中包括 2 个四取代手性中心和 1 个全碳季碳手性中心. 2015 年, 杨震小组 ${ }^{[33]}$ 就采用不对称 Diels-Alder 反应、 Pauson-Khand 反应等构建主要骨架，再通过氧化偶联反 应引入侧链实现了 propindilactone $\mathrm{G}$ 的全合成, 并对原 始结构进行了修正. 而桂敬汉小组 ${ }^{[37]}$ 受生源合成途径 启发, 从甾体内酯 84 出发, 其关键转化包括: (1) Breslow 远端官能团化引入 C-9 位和 C-11 位之间的烯烃; (2) Suárez远端自由基官能团化实现 C-19位选择性氧化; (3) 生物启发的 Wagner-Meerwein 重排实现 B 环扩环; (4) Lewis 酸催化的 C-10 位叔醇手性翻转; (5) 仿生的酯交 换氧杂 Michael 加成串联构建 $5 / 5$ 稠合内酯环系.

如 Scheme 7 所示, 从甾体化合物 84 出发, 采用 Breslow 远端官能团化策略，经过 Mitsunobu 反应，选择 性氯代，接着原位 $\mathrm{E} 2$ 消除得到烯烃 $\mathbf{8 5}$. 之后，将乙酰化 的 85 与三氯异氰酸反应得到关键氯代产物 86. 化合物 86 在 Suárez 条件下，经四氢呋喃中间体，之后被还原得 到 C-19 位羟基, 将羟基转化为易离去基团 OMs 后发生 Wagner-Meerwein 重排, 并被水捕获得到含七元环化合 物 88. 化合物 88 经过 Mukaiyama hydroperoxysilylation 反应，在三氟化硼乙醚的作用下得到内过氧化物，实现 了 C-10 位的手性翻转, 再将五元环内酯 89 还原为二醇, 并用丙酮保护, 经过 Dess-Martin 氧化 C-3 位剩余羟基后 得到 91. 酮 91 羰基 $\alpha$ 位进行区域选择性甲基化，引入两 个甲基，随后发生 Baeyer-Villiger 氧化及 Mukaiyama 脱 氢得到不饱和内酯 92. 还原 92 的过氧键在对甲苯磺酸 作用下发生仿生酯交换/氧杂 Michael 加成串联反应, 实 现了 $5 / 5$ 环系的构建. 接着伯醇 93 发生 Swern 氧化转化 为醛，与 94 反应并消除得到不饱和醛 95.95 发生 Horner-Wadsworth-Emmons 反应得到 $Z$ 式烯烃 97. 随后, 97 发生 Saegusa 氧化得到五元环不饱和酮 98, 在对甲苯 磺酸作用下实现 C-17 位手性翻转. 最终, 烯烃 99 经过 Sharpless 不对称双羟基化，从而实现了 propindilactone $\mathrm{G}$ 的全合成.

\section{3 总结与展望}

综上所述, 丁寒锋小组 ${ }^{[43]}$ 通过两次钛催化的自由 基开环氧反应，构建了 6/7 并环，引入了侧链，同时采用 烯烃复分解反应构建了含有张力的九元环，最终完成了 atro-Schiglautone A 的全合成. Anderson 小组 ${ }^{[42]}$ 采用铑催 


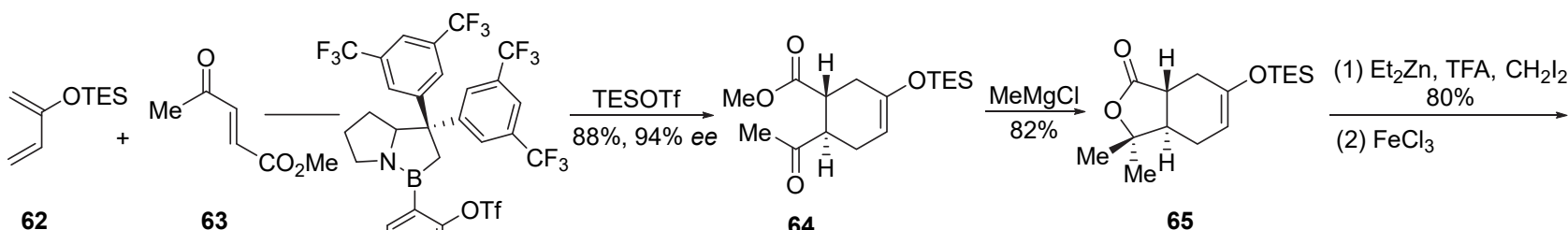

62

63

OTf

64

65

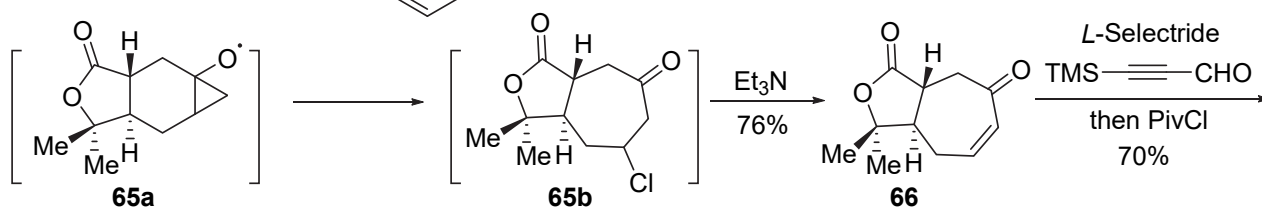

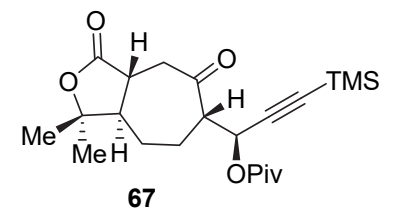

$65 \mathrm{~b} \mathrm{Cl} \quad 66$

$\overbrace{\mathrm{Me}}^{\mathrm{OH}} \frac{\substack{\mathrm{Bu}_{3} \mathrm{SnCH}_{2} \mathrm{l}, \mathrm{NaH} \\ \mathrm{Fe}(\mathrm{dibm})_{3}, 78 \%}}{\mathrm{OH}}$

68

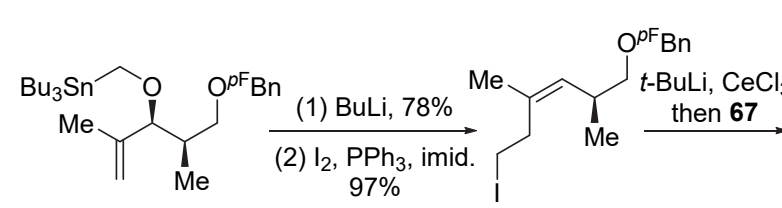

69
70

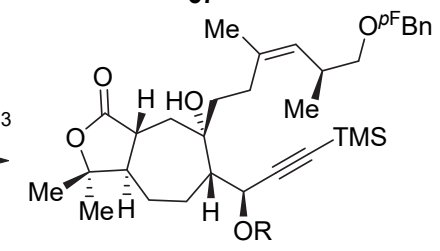

71a: $R=\operatorname{Piv}, 57 \%$ 71b: $R=H, 28 \%$

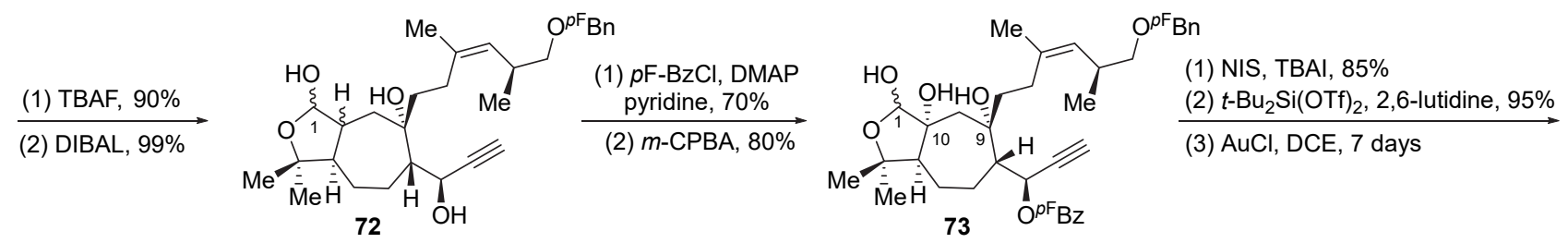

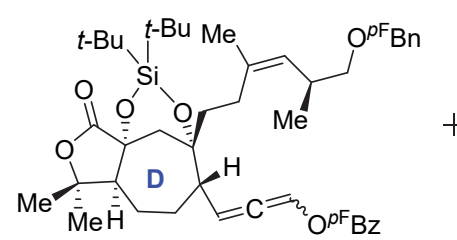

$7452 \%$

$\mathrm{AuCl}, \mathrm{DCE}, 14$ days

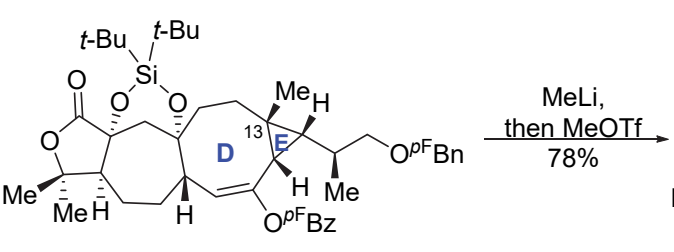
^ $7535 \%$

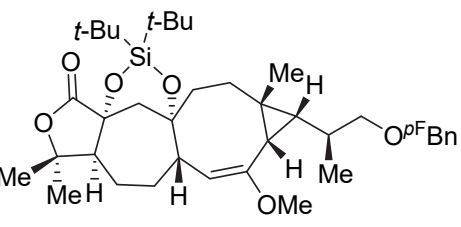

76

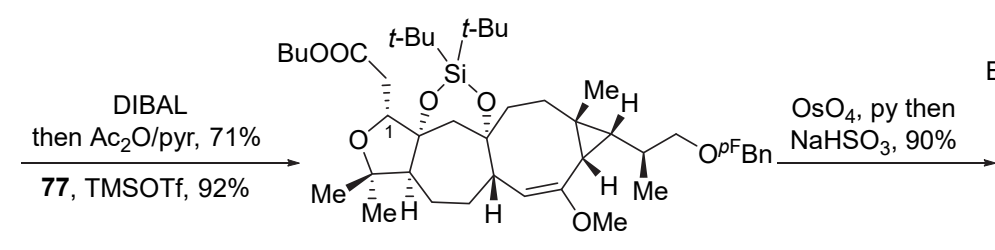

78

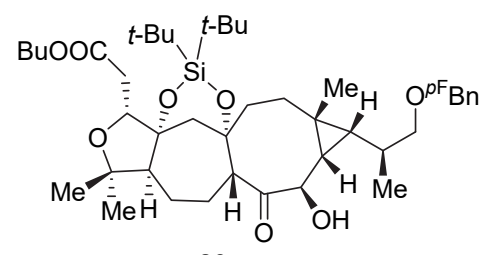

80
(1) Raney-Ni, 79\%

(2) TEMPO, PIDA, 96\% $\left.\mathrm{BuOOC}^{t-\mathrm{Bu}}\right|^{t-\mathrm{Bu}}$<smiles>CCCC[Si]1CCC1=O</smiles>

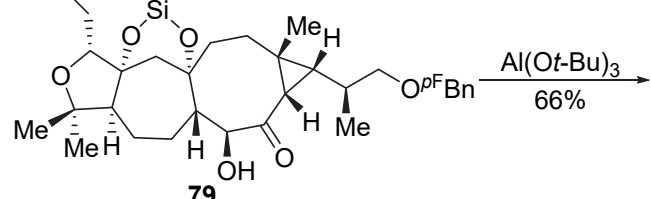<smiles>CCCCOCCO[Si](C)(C(C)(C)C)C(C)(C)C</smiles>

$\mathrm{BuOOC}^{t-\mathrm{Bu}} \mathbf{l}^{t-\mathrm{Bu}}$

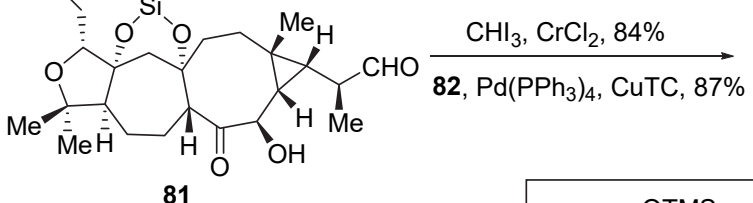

$\mathrm{BuO}_{2} \mathrm{C} t-\mathrm{Bu} v^{t} t-\mathrm{Bu}$

(1) HF, py, then DBU, $94 \%$

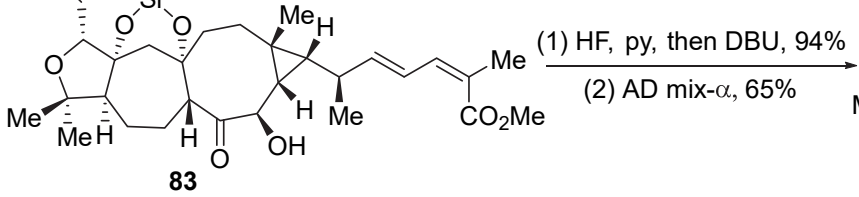

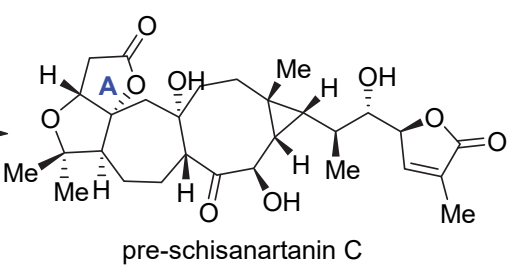

OBu OTMS

图式 6 杨震小组对 pre-schisanartanin $\mathrm{C}$ 的全合成

Scheme 6 Yang's total synthesis of pre-schisanartanin C 


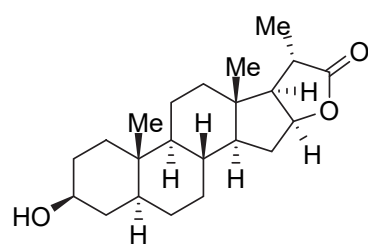

84

\author{
(1) 3-iodobenzoic acid \\ $\mathrm{PPh}_{3}, \mathrm{DEAD}, 87 \%$ \\ (2) $\mathrm{SO}_{2} \mathrm{Cl}_{2}, \mathrm{BPO}$ \\ then $\mathrm{KOH}$
}

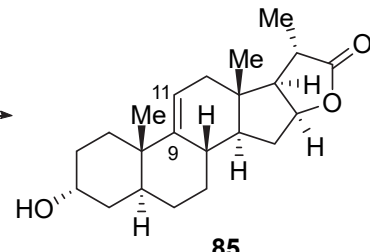

85

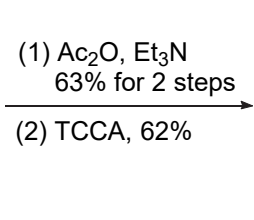

$63 \%$ for 2 steps

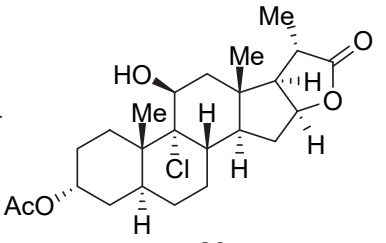

86

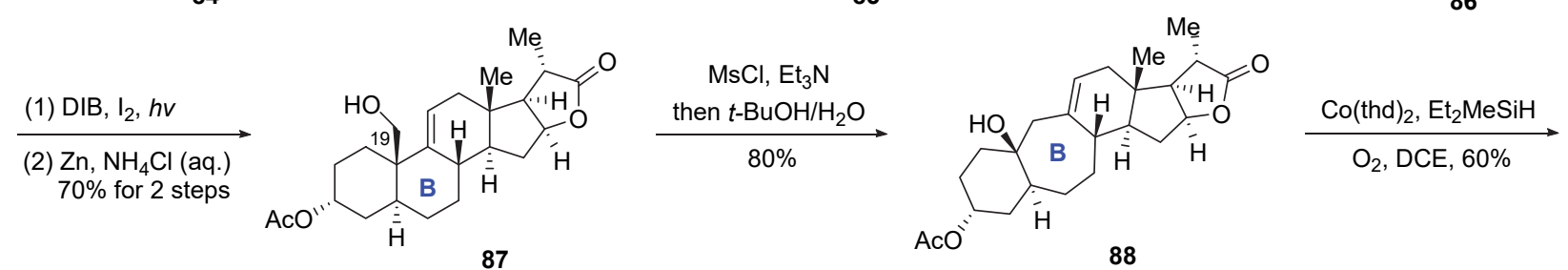

87

88

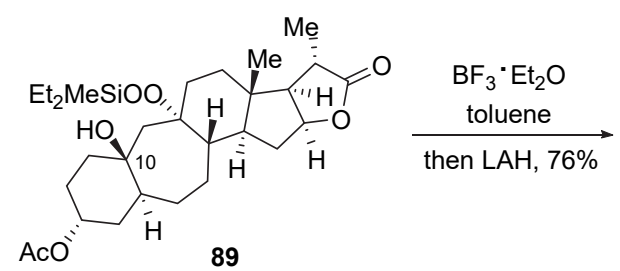

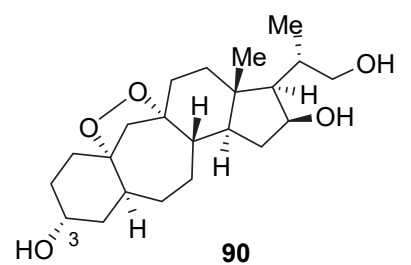

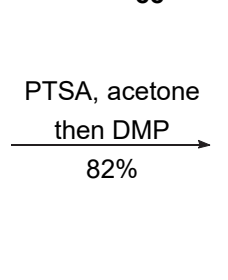

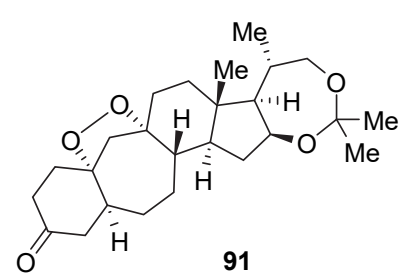

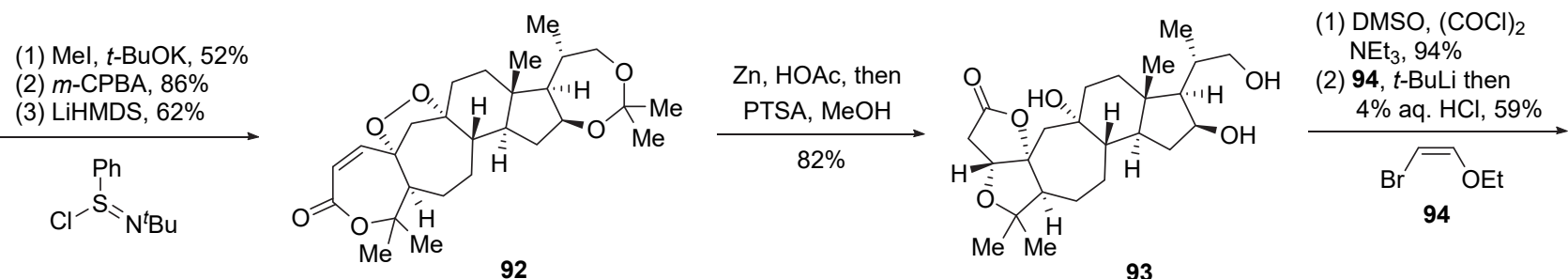

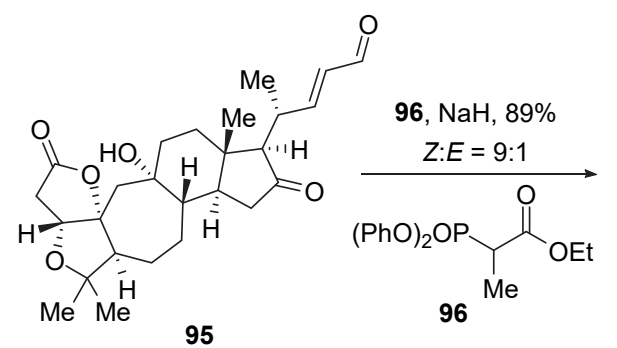

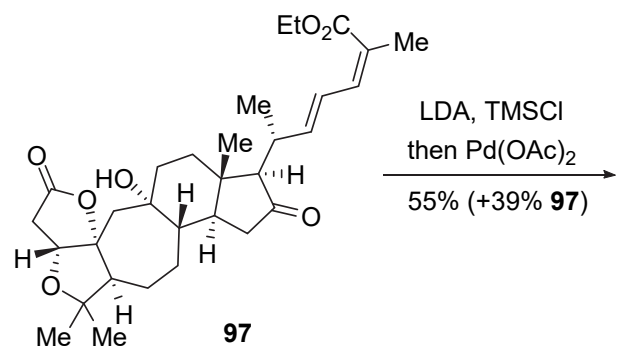

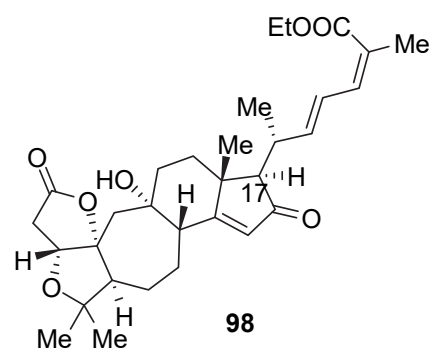
EtOOC

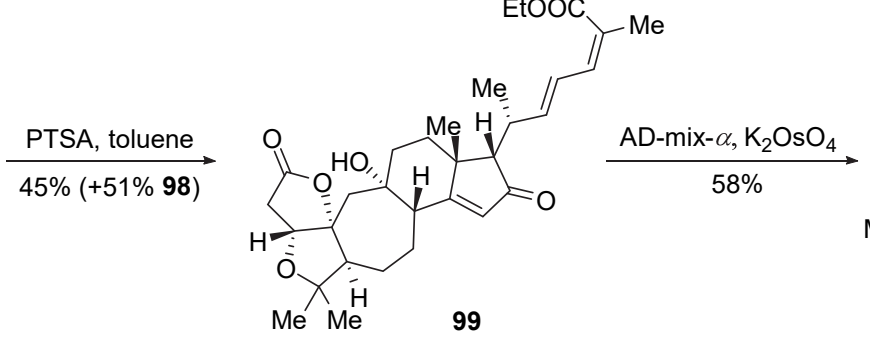

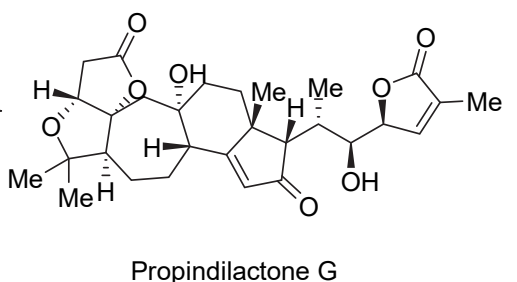

图式 7 桂敬汉小组对 propindilactone $\mathrm{G}$ 的全合成

Scheme 7 Gui's total synthesis of propindilactone G

化 $[2+2+2]$ 策略一步构建了多环结构, 同时汇聚式合 成了 rubriflordilactone B 的多个非对映异构体, 进一步 确定了天然产物结构. 杨震小组 ${ }^{[36]}$ 采用自己发展的不 对称 Diels-Alder 反应为手性全合成铺平了道路, 同时采 用了新策略构建 $5 / 5$ 环系, 巧妙地利用了金催化, 为合 成高张力 $7 / 3$ 环系核心结构提供了新途径, 以 24 步高效 地完成了 pre-schisanartanin $\mathrm{C}$ 的全合成, 并通过单晶确
定了绝对构型. 桂敬汉小组 ${ }^{[37]}$ 则巧妙地采用仿生合成, 从甾体出发, 利用 Wagner-Meerwein 重排和酯交换/氧杂 Michael 加成串联, 构建了七元环和 5/5 环系, 简洁高效 地完成了 propindilactone $\mathrm{G}$ 的全合成工作, 为可能的生 物途径提供了实验依据.

从 2011 年首次全合成报道至今，已经有 6 个小组完 成了 15 个不同的五味子三萜类天然产物及类似物的全 
合成, 对天然产物的结构确定、活性研究及生源途径探 索起到了重要作用. 然而, 五味子三萜类天然产物骨架 类型丰富、数量众多, 而目前全合成主要为五味子降三 萜类天然产物, 并且相关活性研究报道较少. 随着更多 结构类型的五味子三萜类天然产物被分离报道, 期待在 不断涌现的方法学条件下, 可以发展更多快速高效的合 成策略, 从而进行五味子类天然产物全合成及其衍生化 研究, 为其生物测试、药理活性、生源途径研究提供更 多物质基础，进而对药物研发做出更多贡献.

\section{References}

[1] Xiao, W. L.; Li, R. T.; Huang, S. X.; Pu, J. X.; Sun, H. D. Nat. Prod. Rep. 2008, 25, 871.

[2] Shi, Y. M.; Xiao, W. L.; Pu, J. X.; Sun, H. D. Nat. Prod. Rep. 2015, $32,367$.

[3] Tang, Y.; Zhang, Y.; Dai, M.; Luo, T.; Deng, L.; Chen, J.; Yang, Z. Org. Lett. 2005, 7, 885.

[4] Hui, Y. Z. Comprehensive Natural Products in Tradational Chinese Medicine, Shanghai Scientific and Technical Publishers, Shanghai, 2011 (in Chinese)

(惠永正, 中药天然产物大全, 上海科学技术出版社, 上海, 2011.)

[5] Li, R. T.; Zhao, Q. S.; Li, S. H.; Han, Q. Bin; Sun, H. D.; Lu, Y.; Zhang, L. L.; Zheng, Q. T. Org. Lett. 2003, 5, 1023.

[6] Zhang, Y. D.; Tang, Y. F.; Luo, T. P.; Shen, J.; Chen, J. H.; Yang, Z. Org. Lett. 2006, 8, 107.

[7] Zhang, Y. D.; Ren, W. W.; Lan, Y.; Xiao, Q.; Wang, K.; Xu, J.; Chen, J. H.; Yang, Z. Org. Lett. 2008, 10, 665.

[8] Krishnan, K. S.; Smitha, M.; Suresh, E.; Radhakrishnan, K. V. Tetrahedron 2006, 62, 12345.

[9] Fischer, D.; Theodorakis, E. A. Eur. J. Org. Chem. 2007, 25, 4193.

[10] Wang, Q.; Chen, C. Org. Lett. 2008, 10, 1223.

[11] Paquette, L. A.; Kwong, W. L. Org. Lett. 2008, 10, 2111.

[12] Kwong, W. L.; Paquette, L. A. Org. Lett. 2008, 10, 2115.

[13] Paquette, L. A.; Lai, K. W. Org. Lett. 2008, 10, 3781.

[14] Cordonnier, M. C. A.; Jennifer Kan, S. B.; Anderson, E. A. Chem. Commun. 2008, 44, 5818.

[15] Goh, S. S.; Baars, H.; Gockel, B.; Anderson, E. A. Org. Lett. 2012, 14,6278 .

[16] Gockel, B.; Goh, S. S.; Puttock, E. J.; Baars, H.; Chaubet, G.; Anderson, E. A. Org. Lett. 2014, 16, 4480.

[17] Mehta, G.; Bhat, A. B.; Kumara, T. H. S. Tetrahedron Lett. 2009 , $50,6597$.

[18] Mehta, G.; Bhat, B. A.; Suresha Kumara, T. H. Tetrahedron Lett. 2010, 51,4069 .

[19] Mehta, G.; Bhat, B. A. Tetrahedron Lett. 2009, 50, 2474.

[20] Mehta, G.; Yaragorla, S. A. Tetrahedron Lett. 2013, 54, 549.

[21] Matcha, K.; Maity, S.; Malik, C. K.; Ghosh, S. Tetrahedron Lett. 2010, 51,2754

[22] Maity, S.; Matcha, K.; Ghosh, S. J. Org. Chem. 2010, 75, 4192.

[23] Hossain, M. F.; Matcha, K.; Ghosh, S. Tetrahedron Lett. 2011, 52, 6473 .

[24] Bartoli, A.; Chouraqui, G.; Parrain, J. L. Org. Lett. 2012, 14, 122.

[25] Peng, Y.; Duan, S. M.; Wang, Y. W. Tetrahedron Lett. 2015, 56, 4509 .

[26] Wang, Y.; Li, Z.; Lv, L.; Xie, Z. Org. Lett. 2016, 18, 792.

[27] Wang, Y.; Zhang, Y.; Li, Z.; Yang, Z.; Xie, Z. Org. Chem. Front. 2017, 4, 47 .

[28] Werner, B.; Kalesse, M. Org. Lett. 2017, 19, 1524.

[29] Sun, T. W.; Ren, W. W.; Xiao, Q.; Tang, Y. F.; Zhang, Y. D.; Li, Y.; Meng, F. K.; Liu, Y. F.; Zhao, M. Z.; Xu, L. M.; Chen, J. H.; Yang,
Z. Chem. Asian J. 2012, 7, 2321.

[30] Li, Y.; Chen, Z. X.; Xiao, Q.; Ye, Q. D.; Sun, T. W.; Meng, F. K.; Ren, W. W.; You, L.; Xu, L. M.; Wang, Y. F.; Chen, J. H.; Yang, Z. Chem. Asian J. 2012, 7, 2334.

[31] Ren, W. W.; Chen, Z. X.; Xiao, Q.; Li, Y.; Sun, T. W.; Zhang, Z. Y.; Ye, Q. D.; Meng, F. K.; You, L.; Zhao, M. Z.; Xu, L. M.; Tang, Y. F.; Chen, J. H.; Yang, Z. Chem. Asian J. 2012, 7, 2341.

[32] Xiao, Q.; Ren, W. W.; Chen, Z. X.; Sun, T. W.; Li, Y.; Ye, Q. D.; Gong, J. X.; Meng, F. K.; You, L.; Liu, Y. F.; Zhao, M. Z.; Xu, L. M.; Shan, Z. H.; Shi, Y.; Tang, Y. F.; Chen, J. H.; Yang, Z. Angew. Chem., Int. Ed. 2011, 50, 7373.

[33] You, L.; Liang, X. T.; Xu, L. M.; Wang, Y. F.; Zhang, J. J.; Su, Q.; Li, Y. H.; Zhang, B.; Yang, S. L.; Chen, J. H.; Yang, Z. J. Am. Chem. Soc. 2015, 137, 10120.

[34] Han, Y. X.; Jiang, Y. L.; Li, Y.; Yu, H. X.; Tong, B. Q.; Niu, Z.; Zhou, S. J.; Liu, S.; Lan, Y.; Chen, J. H.; Yang, Z. Nat. Commun. 2017, 8,1 .

[35] Liu, D. D.; Sun, T. W.; Wang, K. Y.; Lu, Y.; Zhang, S. L.; Li, Y. H.; Jiang, Y. L.; Chen, J. H.; Yang, Z. J. Am. Chem. Soc. 2017, 139, 5732.

[36] Jiang, Y. L.; Yu, H. X.; Li, Y.; Qu, P.; Han, Y. X.; Chen, J. H.; Yang, Z. J. Am. Chem. Soc. 2020, 142, 573.

[37] Wang, Y.; Chen, B.; He, X.; Gui, J. J. Am. Chem. Soc. 2020, 142, 5007.

[38] Wang, Y.; Chen, B.; He, X.; Gui, J. Chin. J. Chem. 2020, 38, 1339.

[39] Li, J.; Yang, P.; Yao, M.; Deng, J.; Li, A. J. Am. Chem. Soc. 2014, $20,8$.

[40] Yang, P.; Yao, M.; Li, J.; Li, Y.; Li, A. Angew. Chem., Int. Ed. 2016, 55,6964 .

[41] Goh, S. S.; Chaubet, G.; Gockel, B.; Cordonnier, M.-C. A.; Baars, H.; Phillips, A. W.; Anderson, E. A. Angew. Chem., Int. Ed. 2015, $54,12618$.

[42] Mohammad, M.; Chintalapudi, V.; Carney, J. M.; Mansfield, S. J.; Sanderson, P.; Christensen, K. E.; Anderson, E. A. Angew. Chem., Int. Ed. 2019, 58, 18177.

[43] Ma, B.; Zhao, Y.; He, C.; Ding, H. Angew. Chem., Int. Ed. 2018, 57, 15567.

[44] Wang, H.; Zhang, X.; Tang, P. Chem. Sci. 2017, 8, 7246

[45] Wang, H.; Wang, L.; Li, Y.; Zhang, X.; Tang, P. Chin. J. Chem. 2019, 37, 255.

[46] Wang, L.; Wang, H.; Li, Y.; Tang, P. Angew. Chem., Int. Ed. 2015, 54,5732 .

[47] Li, X.; Cheong, P. H. Y.; Carter, R. G. Angew. Chem., Int. Ed. 2017, $56,1704$.

[48] Yan, B.; Hu, K.; Sun, H.; Puno, P. Chin. J. Org. Chem. 2018, 38, 2259 (in Chinese).

(颜秉超，胡坤，孙汉董，普诺・白玛丹增，有机化学， 2018，38， 2259.)

[49] Yang, Z. Acc. Chem. Res. 2019, 52, 480.

[50] Bhat, B. A.; Rashid, S.; Sengupta, S.; Mehta, G. Asian J. Org. Chem. 2020, 9, 449 .

[51] Fu, S.; Liu, B. Org. Chem. Front. 2020, 7, 1903.

[52] Meng, F. Y.; Sun, J. X.; Li, X.; Yu, H. Y.; Li, S. M.; Ruan, H. L. Org. Lett. 2011, 13, 1502.

[53] Xiao, W. L.; Yang, L. M.; Gong, N. B.; Wu, L.; Wang, R. R.; Pu, J. X.; Li, X. L.; Huang, S. X.; Zheng, Y. T.; Li, R. T.; Lu, Y.; Zheng, Q. T.; Sun, H. D. Org. Lett. 2006, 8, 991.

[54] Yang, P.; Li, J.; Sun, L.; Yao, M.; Zhang, X.; Xiao, W. L.; Wang, J. H.; Tian, P.; Sun, H. D.; Puno, P. T.; Li, A. J. Am. Chem. Soc. 2020, 142,13701 .

[55] Lei, C.; Xiao, W. L.; Huang, S. X.; Chen, J. J.; Pu, J. X.; Sun, H. D. Tetrahedron 2010, 66, 2306.

[56] Lei, C.; Huang, S. X.; Chen, J. J.; Yang, L. Bin; Xiao, W. L.; Chang, Y.; Lu, Y.; Huang, H.; Pu, J. X.; Sun, H. D. J. Nat. Prod. 2008, 71,1228 . 